July 1999

UT-KOMABA/99-9

cond-mat/9907335

\title{
Loop Model with Generalized Fugacity in Three Dimensions
}

\author{
Saburo Higuchi周 \\ Department of Pure and Applied Sciences, \\ The University of Tokyo, Komaba \\ Komaba, Meguro, Tokyo 153-8902, Japan
}

\begin{abstract}
A statistical model of loops on the three-dimensional lattice is proposed and is investigated. It is $\mathrm{O}(n)$-type but has loop fugacity that depends on global three-dimensional shapes of loops in a particular fashion. It is shown that, despite this non-locality and the dimensionality, a layer-to-layer transfer matrix can be constructed as a product of local vertex weights for infinitely many points in the parameter space. Using this transfer matrix, the site entropy is estimated numerically in the fully packed limit.
\end{abstract}

PACS: 05.20.-y 05.40.Fb 05.50.+q 36.20.-r

Keywords: self-avoiding walk; random walk; $\mathrm{O}(n)$ model; loop model; polymer

${ }^{*}$ e-mail: hig@rice.c.u-tokyo.ac.jp 


\section{Introduction}

Loop models are interesting examples of statistical models of extended objects. They are related to the $\mathrm{O}(n)$ spin model [1, 2], a surface growth model [3], the protein folding problem [4], and so on. It includes the fully packed loop model [5] and the Hamiltonian cycle problem [6, 8, 8] as particular limits.

The partition function of an $\mathrm{O}(n)$ loop model on a lattice with $N$ sites at the inverse temperature $x$ is given by

$$
Z_{\text {loop }}\left(n, x^{-1}\right)=\sum_{c \in \mathcal{C}} x^{\mathcal{N}_{\mathrm{S}}(c)-N} n^{\mathcal{N}_{\mathrm{L}}(c)} \quad(n, x \in \mathbb{R}) .
$$

The summation is taken over the set $\mathcal{C}$ of all the non-intersecting loop configurations drawn along links of the lattice. The integers $\mathcal{N}_{\mathrm{L}}(c)$ and $\mathcal{N}_{\mathrm{S}}(c)$ denote the number of loops and of sites visited by loops.

One may hope to study the model (11) by the transfer matrix approach. For $n \in \mathbb{Z}_{+}$, this is done in a simple way; one introduces link variables whose values are either occupied states with one of $n$ colors or an unoccupied state and lets them interact on sites. A transfer matrix is written as a product of vertex weights straightforwardly.

For $n \notin \mathbb{Z}_{+}$, however, the partition sum (11) cannot be rewritten in terms of local degrees of freedom such as link variables in a simple way. It is not trivial to have a local transfer matrix 1 . I say a transfer matrix is local when its component is written as a product of weights each of which is determined by the local state configuration around a lattice site.

It is surprising that, in two dimensions, $n \notin \mathbb{Z}_{+}$models admit a mapping onto a state sum model with a local vertex weight and thus have local transfer matrices [9]. In fact, by choosing $s \in \mathbb{C}$ satisfying $n=s+s^{-1}, Z_{\text {loop }}\left(n, x^{-1}\right)$ can be written as

$$
\begin{aligned}
Z_{\text {loop }}\left(n, x^{-1}\right) & =\sum_{c \in \mathcal{C}} x^{\mathcal{N}_{\mathrm{S}}(c)-N}\left(s+s^{-1}\right)^{\mathcal{N}_{L}(c)} \\
& =\sum_{c \in \overline{\mathcal{C}}} x^{\mathcal{N}_{\mathrm{S}}(c)-N} \prod_{L \in \overline{\mathcal{L}}(c)} s^{ \pm 1}
\end{aligned}
$$

where $\overline{\mathcal{C}}$ is the set of loop configurations with a direction associated with each loop. The set $\overline{\mathcal{L}}(c)$ consists of all the directed loops in a configuration $c$. A loop with the (counter-)clockwise direction is given a weight $s^{+1}\left(s^{-1}\right)$. This weight can be realized by associating $s^{+1 / 4}\left(s^{-1 / 4}\right)$ with each right(left)-turn site and the model can be regarded as a state sum model with a local vertex weight. This trick has made the study of two-dimensional loop models very fruitful.

Physics of loops in three dimensions is very attractive. It is not only realistic in the context of condensed matter physics but also has rich mathematical structures. For instance, loops can be knotted or linked only in three dimensions 10 . There has been a continuous suspicion that two-dimensional models of loops

\footnotetext{
${ }^{1}$ The use of the connectivity basis is discussed in subsection 5.4 .
} 
has missed some important ingredient in real physics, e.g. the protein folding problem.

The analysis of loop models in higher dimensions is, however, extremely hard to perform. Needless to say, the number of configurations increases considerably. Moreover, specialties of two dimensions cannot be used to simplify problems any more. Note that the mapping (2) makes use of the fact that the a directed loop turns around either clockwisely or counter-clockwisely just once. One may think that this kind of trick never works in higher dimensions.

In this article, I propose a model which generalizes (1) in a fashion specific to three dimensions. It is furnished with loop fugacity that depends on the global three-dimensional shape of loops. I show that, despite this generalization which makes the model even more non-local, a local transfer matrix for the system can be constructed for a number of choices of fugacity. These choices include the one that gives zero or non-integer weight to loops.

This article is organized as follows. In section 2, I define a loop model in three dimensions generalizing (11). Its local transfer matrix is constructed for a family of points in the parameter space in section 3. In section 1 , this transfer matrix is numerically diagonalized to estimate the site entropy in the fully packed limit $x^{-1} \rightarrow 0$. In the last section 5 , I discuss my results and their relation to combinatorial problems. In an appendix, a technical issue on the block-diagonalization of the transfer matrix is addressed.

\section{Generalized fugacity}

I define a statistical model of loops on the three-dimensional simple cubic lattice $\mathbb{Z}^{3}=\left\{\sum_{i=1}^{3} m_{i} \mathbf{e}_{i} \in \mathbb{R}^{3} \mid m_{i} \in \mathbb{Z}\right\}, \mathbf{e}_{i} \cdot \mathbf{e}_{j}=\delta_{i j}$. The partition function is given by

$$
Z_{\text {loop }}[n]\left(x^{-1}\right)=\sum_{c \in \mathcal{C}} x^{\mathcal{N}_{\mathrm{S}}(c)-N} \prod_{L \in \mathcal{L}(c)} n(A(L)),
$$

where $\mathcal{L}(c)$ is the set of loops in a configuration $c$. The loop fugacity $n$ is now promoted to a function which depends on the shape of $L \in \mathcal{L}(c)$ through a quantity $A(L) \in \mathbb{R}$ defined below.

To define $A(L)$, one begins with associating a closed trajectory on the unit sphere with each loop $L$. One picks a direction for $L$. On every point $\mathrm{x} \in L \subset \mathbb{R}^{3}$ except for sites where $L$ makes a turn, there is a unit tangential vector $\mathbf{v}(\mathbf{x})$ to $L$; it is either of $\pm \mathbf{e}_{i}, i=1,2,3$. One may regard $\mathbf{v}(\mathbf{x})$ as a mapping from $L \backslash$ ('turn-sites') to the unit sphere $\mathrm{S}^{2}$.

As one walks along $L, \mathbf{v}(\mathbf{x})$ jumps from a point to another on $\mathrm{S}^{2}$. One can naturally interpolate these points to define a continuous trajectory $\mathbf{v}: L \rightarrow \mathrm{S}^{2}$. One has only to declare that $\mathbf{v}(\mathbf{x})$ moves along the geodesic (of length $\frac{1}{2} \pi$ ) on $\mathrm{S}^{2}$ at each turn-site. This is equivalent with smoothing a loop in neighborhoods of turn-sites keeping the loop within a plane (Fig.11). Then one defines $A(L)$ to be the oriented area encircled in the right of the trajectory $\mathbf{v}(\mathbf{x})$. On the lattice 

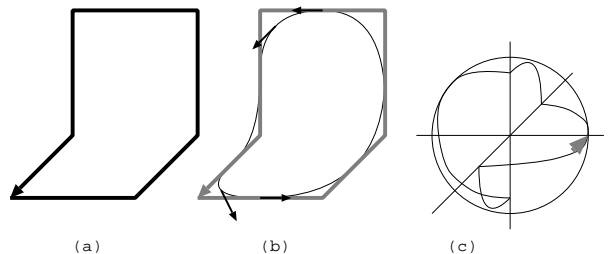

(c)

Figure 1: The definition of $A(L)$. (a) The original loop $L$ with a direction associated. (b) The loop smoothed at turn-sites. (c) Trajectory of the tangent vector on the unit sphere. An area of $A=2 \pi$ is enclosed.

$\mathbb{Z}^{3}, A(L)$ takes values

$$
A(L)=\frac{1}{2} m \pi(m \in \mathbb{Z}) .
$$

In two dimensions, the quantity $A(L)$ takes values $\pm 2 \pi$ and this signature corresponds to that in (2). Therefore (3) incorporates an essential ingredient of three dimensional loops and regarded as a natural generalization of (2).

As is evident from the above construction, there is certain ambiguity in $A(L)$. First, because the trajectory is drawn on a closed surface of area $4 \pi$, $A(L)$ is well-defined up to $4 \pi$. Second, the signature of $A(L)$ can be changed when the picked direction of $L$ is reversed. I require that $n(\cdot)$ in (3) absorbs this ambiguity. Hence it should satisfy

$$
n(-A)=n(A)
$$

and

$$
n(A)=n(A+4 \pi) .
$$

Eqs. (可), (6) and (值) imply that the fugacity function $n(\cdot)$ can be specified by five parameters $n(A), A=0, \frac{1}{2} \pi, \pi, \frac{3}{2} \pi, 2 \pi$.

In spite of the above restriction on $n(A)$, the model (3) includes interesting cases. Consider, for example, fugacity

$$
n(A)=n_{0} \delta_{0}^{(4)}(A),
$$

with

$$
\delta_{b}^{(a)}(A)= \begin{cases}1 & \text { if }\left(\frac{A}{\pi}-b\right) \equiv 0 \bmod a \\ 0 & \text { otherwise }\end{cases}
$$

The sum in (3) is then restricted to configurations which consist only of loops with the oriented area $A \equiv 0 \bmod 4 \pi$. It should be interesting to compare the site entropy with that of the model with $n(A)=n_{0}$. It is also tempting to ask whether such an additional constraint changes the critical behavior or not. The present case reminds one of the fully packed loop model in two dimensions. Its universality class differs from that of densely packed loop phase when the one imposes additional constraint that the loop length must be even [9, 11. 


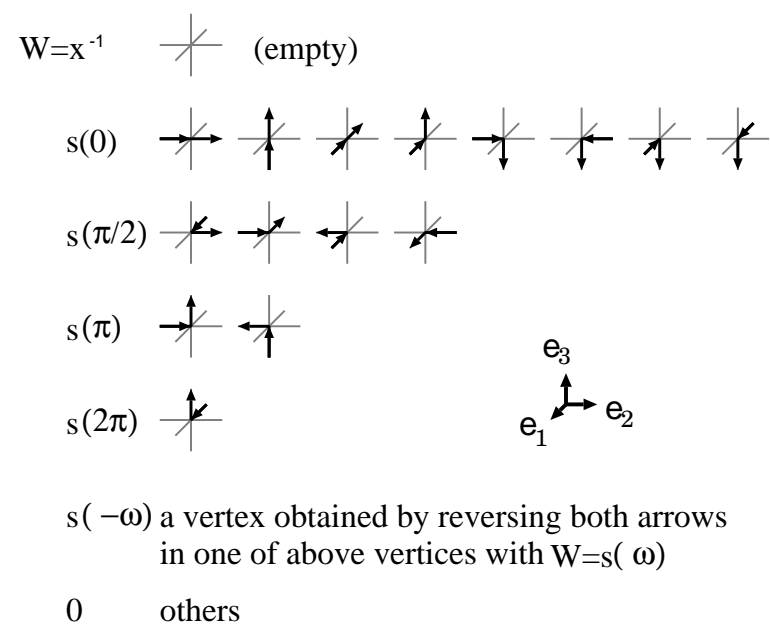

Figure 2: Vertex weights $W$ for $Z_{\text {vertex }}[s]\left(x^{-1}\right)$.

\section{Transfer matrices from local vertex weights}

In order to construct a local layer-to-layer transfer matrix, I define a vertex model and show that it is equivalent with (3).

The local degree of freedom $z$ of the vertex model lives on each link $\left\langle\mathbf{r}, \mathbf{r} \pm \mathbf{e}_{i}\right\rangle$. It takes one of three values $\leftarrow, \rightarrow$, and - (empty). On each site, six neighboring link variables interact by the vertex weight $W$ defined in Fig. 2, where $s(\omega)$ is a function that satisfies

$$
s\left(A_{1}\right) \times s\left(A_{2}\right)=s\left(A_{1}+A_{2}\right)
$$

and is specified further below. The partition function of the vertex model is

$$
Z_{\text {vertex }}[s]\left(x^{-1}\right)=\sum_{z=\leftarrow, \rightarrow,-} \prod_{\mathbf{r} \in \mathbb{Z}^{3}} W\left(\left\{z\left(\left\langle\mathbf{r}, \mathbf{r} \pm \mathbf{e}_{i}\right\rangle\right)\right\}\right) .
$$

Evidently, the partition function (10) has a local transfer matrix which is a product of $W$ 's. Now I show that (10) is equivalent with (3) for an appropriate $s$.

Because the weight $W$ is nonzero only when there is one incoming and one outgoing arrow, contribution to the partition sum (10) comes only from the set $\overline{\mathcal{C}}$ of directed loop configurations

$$
\begin{aligned}
Z_{\text {vertex }}[s]\left(x^{-1}\right) & =\sum_{c \in \overline{\mathcal{C}}} x^{\mathcal{N}_{L}(c)-N} \prod_{L \in \overline{\mathcal{L}}(c)} X(L), \\
X(L) & =\prod_{\mathbf{r} \in L \cap \mathbb{Z}^{3}} W\left(\left\{z\left(\left\langle\mathbf{r}, \mathbf{r} \pm \mathbf{e}_{i}\right\rangle\right)\right\}\right)=\prod_{\mathbf{r} \in L \cap \mathbb{Z}^{3}} s(\omega(\mathbf{r})),
\end{aligned}
$$


where $s(\omega(\mathbf{r}))$ is the weight for the vertex at $\mathbf{r} \in \mathbb{Z}^{3}$ in Fig.2.

Let us inspect the contribution $X(L)$. It is crucial to observe that

$$
A(L)=\sum_{\mathbf{r} \in L \cap \mathbb{Z}^{3}} \omega(\mathbf{r}) .
$$

Actually, the weight system in Fig.2 is designed to have this property in refs. 112, 13, 14]. in the context of random walk with a spin factor [15, 16]. By eqs.(9) and (13), contribution from a loop $L$ becomes

$$
X(L)=s(A(L)) .
$$

Rewriting (11) as a sum over undirected loops, as was done for $\mathrm{O}(n)$ loop model, one arrives at

$$
Z_{\text {vertex }}[s]\left(x^{-1}\right)=\sum_{c \in \mathcal{C}} x^{\mathcal{N}_{L}(c)-N} \prod_{L \in \mathcal{L}(c)}(s(A(L))+s(-A(L))) .
$$

Thus, if one can write $n(A)$ in (3) as

$$
n(A)=s(A)+s(-A)
$$

with a function $s$ which satisfies (9), $Z_{\text {loop }}[n]\left(x^{-1}\right)$ is equal to $Z_{\text {vertex }}[s]\left(x^{-1}\right)$.

The requirement (9) together with the restriction (5),(6) and (蛋) on $n(A)$ requires $s$ to have a simple form

$$
s(A)=e^{i J A}
$$

with $J \in \mathbb{Z} / 2$. It is enough to consider the cases $J=0, \frac{1}{2}, 1, \frac{3}{2}$ and 2 because of (14) [12]. Hereafter, a shorthand notation

$$
Z_{J}\left(x^{-1}\right):=Z_{\mathrm{loop}}\left[n(\cdot)=e^{i J A(\cdot)}+e^{-i J A(\cdot)}\right]\left(x^{-1}\right)
$$

is used.

The vertex weights at $J=0$ and $J=2$ enjoy a special property $s(\omega)=$ $s(-\omega)$. This fact enables one to define a model with only two microscopic states $\leftrightarrow$ and - :

$$
\begin{aligned}
\bar{Z}_{\mathrm{vertex}}[s]\left(x^{-1}\right): & =\sum_{z=\leftrightarrow,-} \prod_{\mathbf{r} \in \mathbb{Z}^{3}} W\left(\left\{z\left(\left\langle\mathbf{r}, \mathbf{r} \pm \mathbf{e}_{i}\right\rangle\right)\right\}\right) \\
& =\sum_{c \in \mathcal{C} \cap \mathbb{Z}^{3}} x^{\mathcal{N}_{L}(c)-N} \prod_{L \in \mathcal{L}(c)} s(A(L)) .
\end{aligned}
$$

I denote them by $Z_{0^{\prime}}$ and $Z_{2^{\prime}}$ and refer them as 'primed $J$.'

The fugacity functions corresponding to $J=0, \frac{1}{2}, 1, \frac{3}{2}, 2,0^{\prime}$ and $2^{\prime}$ are listed in Table 1. They indeed give $n(A) \leq 0$ or $n(A) \notin \mathbb{Q}$ for some loops.

Although only finite number of vertex models $J=0, \frac{1}{2}, 1, \frac{3}{2}, 2,0^{\prime}$ and $2^{\prime}$ are constructed in the above, it is possible to construct an infinite number of 


\begin{tabular}{|c|l|}
\hline$J$ & $n(\cdot)$ \\
\hline \hline 0 & $2 \delta_{0}^{\left(\frac{1}{2}\right)}(\equiv 2)$ \\
$\frac{1}{2}$ & $2\left(\delta_{0}^{(4)}-\delta_{2}^{(4)}\right)+\sqrt{2}\left(\delta_{\frac{1}{2}}^{(4)}+\delta_{\frac{7}{2}}^{(4)}-\delta_{\frac{3}{2}}^{(4)}-\delta_{\frac{5}{2}}^{(4)}\right)$ \\
1 & $2\left(\delta_{0}^{(2)}-\delta_{1}^{(2)}\right)$ \\
$\frac{3}{2}$ & $2\left(\delta_{0}^{(4)}-\delta_{2}^{(4)}\right)-\sqrt{2}\left(\delta_{\frac{1}{2}}^{(4)}+\delta_{\frac{7}{2}}^{(4)}-\delta_{\frac{3}{2}}^{(4)}-\delta_{\frac{5}{2}}^{(4)}\right)$ \\
2 & $2\left(\delta_{0}^{(1)}-\delta_{\frac{1}{2}}^{(1)}\right)$ \\
\hline $0^{\prime}$ & $\delta_{0}^{\left(\frac{1}{2}\right)}\left(\equiv 1^{(1)}\right.$ \\
$2^{\prime}$ & $\delta_{0}^{(1)}-\delta_{\frac{1}{2}}^{(1)}$ \\
\hline
\end{tabular}

Table 1: The generalized fugacity $n$ which generates the semigroup of allowed models. The function $\delta_{b}^{(a)}(\cdot)$ is defined in eq.(8).

ones by taking the direct sum of the space of their microscopic states. More precisely, one generalizes the link variable to take one of $2 q+1\left(q \in \mathbb{Z}_{+}\right)$states: $\leftarrow_{k}, \rightarrow_{k}$ with one the $k$-th color $(k=1, \ldots, q)$ and an uncolored empty state - . Introducing parameters $J_{k},\left(1 \leq k \leq q, J_{k} \in\left\{0, \frac{1}{2}, 1, \frac{3}{2}, 2\right\}\right)$ the vertex weight assignments in Fig.2 are supplemented by additional rules:

- If the both two arrows have the $k$-th color, then $W==e^{i J_{k} \omega}$

- If the two colors do not agree, $W=0$.

The cases $J_{k}=0^{\prime}, 2^{\prime}$ are handled in an obvious manner.

The fugacity of this 'direct sum' model $Z_{J_{1} \oplus J_{2} \oplus \cdots \oplus J_{q}}$ is the sum of those for $Z_{J_{1}}, \ldots, Z_{J_{q}}$.

$$
\begin{aligned}
& n(A)=\sum_{k=1}^{q}\left[\left(e^{i J_{k} A}+e^{-i J_{k} A}\right) \times B\left(J_{k}\right)\right], \\
& B(J)= \begin{cases}1 & \left(J=0, \frac{1}{2}, 1, \frac{3}{2}, 2\right), \\
\frac{1}{2} & \left(J=0^{\prime}, 2^{\prime}\right) .\end{cases}
\end{aligned}
$$

One immediately notices that

$$
Z_{0^{\prime} \oplus 0^{\prime}}=Z_{0}, \quad Z_{2^{\prime} \oplus 2^{\prime}}=Z_{2} .
$$

Thus the fugacity functions expressible via vertex models form an infinite semigroup under addition 7 generated by $J=0^{\prime}, \frac{1}{2}, 1, \frac{3}{2}$ and $2^{\prime}$.

One can also take the 'tensor product' of the space of microscopic states of $Z_{J_{1}}$ and $Z_{J_{2}}$ to define a model $Z_{J_{1} \otimes J_{2}}$. Let the link variable take five values

${ }^{2}$ This direct sum operation may be used for the lattice construction [12, 13, 14]. of higher-spin three-dimensional field theories. 
$\left(z_{1}, z_{2}\right)=\uparrow \uparrow, \uparrow \downarrow, \downarrow \uparrow, \downarrow \downarrow$, and $\|$. The vertex weight $W$ is defined to be the product of $W$ 's with $J=J_{1}$ and $J=J_{2}$. Then the loop fugacity becomes

$$
n(A)=\left[\left(e^{i J_{1} A}+e^{-i J_{1} A}\right) \times B\left(J_{1}\right)\right] \times\left[\left(e^{i J_{2} A}+e^{-i J_{2} A}\right) \times B\left(J_{2}\right)\right] .
$$

However, the partition function $Z_{J_{1} \otimes J_{2}}$ is equivalent with an appropriate direct sum

$$
Z_{J_{1} \otimes J_{2}}=Z_{\bar{J}_{1} \oplus \cdots \oplus \bar{J}_{q}}
$$

corresponding to composition rule of the representation of $\mathrm{SU}(2)$ and does not provide a new fugacity function.

\section{Entropy estimates}

I numerically diagonalize the transfer matrices constructed in section 3 . Throughout this section, I concentrate on the fully packed limit $x^{-1} \rightarrow 0$ where all the sites are visited by a loop. This simple case is in fact very interesting case; in two-dimensions, this limit yields a new universality class with a shifted central charge on several bipartite lattices and has been attracting much attention [11, 17, 18, 19]. It also constitutes an interesting combinatorial problem when the the two strong constraints are combined: the fully-packing constraint $x^{-1}$ and the one like (7). One may speculate that the system becomes more frustrated.

The site entropy in the thermodynamic limit is defined by

$$
f[n](\infty)=\lim _{N \rightarrow \infty} \frac{1}{N} \log Z_{\text {loop }}[n]\left(x^{-1}=0\right) .
$$

I evaluate this quantity on quasi-one-dimensional geometry by calculating the largest eigenvalue of the transfer matrix in an appropriate sector.

Let $T$ be the layer-to-layer transfer matrix in $+\mathbf{e}_{3}$ (vertical) direction for the vertex model defined in (10). If one takes a lattice of size $L_{1} \times L_{2}$ in the $\mathbf{e}_{1}$ and $\mathbf{e}_{2}$ (horizontal) directions, $T$ acts on linear combinations of arrays of $L_{1} \times L_{2}$ vertical (colored) arrows. One can take either hard-wall or periodic boundary condition in the horizontal directions.

It is important to note that the transfer matrix $T$ commutes with the operator giving the net flow of arrows of $k$-th color in $+\mathbf{e}_{3}$ direction

$$
d_{k}=\left(\# \uparrow_{k}\right)-\left(\# \downarrow_{k}\right),
$$

which is understood as

$$
d_{k}=\left(\# \uparrow_{k}\right) \bmod 2
$$

for $J_{k}=0^{\prime}$ and $2^{\prime}$. Thus $T$ is block-diagonalized as

$$
T=\bigoplus_{\mathbf{d}} T_{\mathbf{d}}, \quad \mathbf{d}=\left(d_{1}, \ldots, d_{q}\right) .
$$




\begin{tabular}{|c|c|c|c|c|c|c|}
\hline $\bar{J}$ & $2 \times 2(\mathrm{~h})$ & $3 \times 3(\mathrm{~h})$ & $3 \times 4(\mathrm{~h})$ & $2 \times 2(\mathrm{p})$ & $3 \times 3(\mathrm{p})$ & $3 \times 4(p)$ \\
\hline$\overline{00}$ & 0.54202495 & 0.59145447 & 0.63524092 & $\bar{~} 1.0585126$ & 0.83841678 & 0.83340128 \\
\hline$\frac{1}{2}$ & 0.27123680 & 0.33576248 & 0.35050951 & 0.79451346 & 0.55900063 & 0.55895924 \\
\hline 1 & 0.51585927 & 0.50234791 & 0.55646223 & 0.97170402 & 0.69812631 & 0.69832061 \\
\hline$\frac{3}{2}$ & 0.35592318 & 0.35908194 & 0.37136801 & 0.79451346 & 0.57435935 & 0.49496387 \\
\hline 2 & 0.49499647 & 0.45468972 & 0.51615498 & 1.0406166 & 0.66200716 & 0.67440981 \\
\hline$\overline{0^{\prime}}$ & 0.46298939 & 0.55650697 & 0.60072954 & 0.91847381 & 0.79631788 & 0.80135760 \\
\hline $2^{\prime}$ & 0.38697370 & 0.37695844 & 0.42272584 & 0.87898824 & 0.55608904 & 0.58497412 \\
\hline & $4 \times 4 \times 4(p)$ & & & & & \\
\hline$\overline{0^{\prime}}$ & 0.81947983 & & & & & \\
\hline $2^{\prime}$ & 0.60931946 & & & & & \\
\hline
\end{tabular}

Table 2: The site entropy estimated numerically. $L_{1} \times L_{2}$ is the size of a layer while (p) and (h) mean periodic and hard-wall boundary conditions within a layer.

The quantity (24) is related to the largest eigenvalue in the sector $\mathbf{d}=\mathbf{0}$ by

$$
f[n](\infty)=\lim _{L_{1}, L_{2} \rightarrow \infty} \frac{1}{L_{1} L_{2}} \log \left|\lambda_{\mathbf{0}}^{0}\left(L_{1}, L_{2}\right)\right|,
$$

where $\lambda_{\mathbf{d}}^{i}\left(L_{1}, L_{2}\right)$ is the $i$-th largest eigenvalue of $T_{\mathbf{d}}\left(L_{1}, L_{2}\right)$. The condition $\mathbf{d}=\mathbf{0}$ excludes the configurations that have unbalanced arrows traveling along the infinite direction.

I have obtained the largest eigenvalue of the block $T_{\mathbf{0}}$ via the standard algorithm for sparse eigenproblems. The finite- $L_{1}, L_{2}$ results are shown in Tables 2 and 3 .

The obstacle in making $L_{1}$ and $L_{2}$ large in the actual numerical work is of course the exponential growth of the dimensionality of the transfer matrix. The selection of $\mathbf{d}=\mathbf{0}$ sector helps to reduce the dimensionality, though the improvement is polynomial. For $J=0^{\prime}$ and $2^{\prime}$, one saves only the factor of two,

$$
\operatorname{dim}(\mathbf{d}=\mathbf{0} \text { sector }) \sim 2^{L_{1} L_{2}} \times \frac{1}{2} \text { as } L_{1} L_{2} \rightarrow \infty,
$$

while for other (unprimed) values,

$$
\operatorname{dim}(\mathbf{d}=\mathbf{0} \text { sector }) \sim 3^{L_{1} L_{2}} \times\left(\frac{3}{4 \pi L_{1} L_{2}}\right)^{1 / 2} \text { as } L_{1} L_{2} \rightarrow \infty .
$$

For the direct sum $Z_{\oplus_{k} J_{k}}$ with $p$ primed $J$ 's and $u$ unprimed $J$ 's, it becomes

$$
\operatorname{dim}(\mathbf{d}=\mathbf{0} \text { sector }) \sim(1+2 u+p)^{L_{1} L_{2}} \times 2^{-p}\left(\frac{3}{4 \pi L_{1} L_{2}}\right)^{u / 2}
$$

\footnotetext{
${ }^{3}$ I have also measured several leading eigenvalues of $T_{\mathbf{d}}$ with $\mathbf{d}=\left(d_{1}, \ldots, d_{q}\right), d_{k}=0, \pm 1$. These are related to correlation length of operators in the theory. These results will be reported elsewhere.
} 


\begin{tabular}{|c|c|c|c|c|c|}
\hline$\oplus_{k} J_{k}$ & $n$ & $2 \times 2(\mathrm{~h})$ & $3 \times 3(\mathrm{~h})$ & $2 \times 2(\mathrm{p})$ & $3 \times 3(\mathrm{p})$ \\
\hline 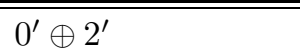 & $2 \delta_{0}^{(1)}$ & 0.52330515 & 0.57390934 & 1.0502400 & 0.81503626 \\
\hline $0 \oplus 0$ & 4 & 0.64498133 & 0.65020710 & 1.2354255 & 0.90747958 \\
\hline $0 \oplus 2$ & $4 \delta_{0}^{(1)}$ & 0.63331428 & 0.62765833 & 1.232054 & 0.88008432 \\
\hline $0^{\prime} \oplus 2^{\prime} \oplus 1$ & $4 \delta_{0}^{(2)}$ & 0.63216104 & 0.61664018 & 1.2110124 & 0.86121744 \\
\hline $0 \oplus 0 \oplus 0 \oplus 0$ & 8 & 0.76911076 & 1.4501153 & 1.0108338 & \\
\hline $0 \oplus 0 \oplus 2 \oplus 2$ & $8 \delta_{0}^{(1)}$ & 1.3280134 & 1.4490048 & 0.98982172 & \\
\hline $0 \oplus 1 \oplus 1 \oplus 2$ & $8 \delta_{0}^{(2)}$ & 0.75985245 & 1.4364447 & 0.97947213 & \\
\hline $0^{\prime} \oplus 2^{\prime} \oplus \frac{1}{2} \oplus 1 \oplus \frac{3}{2}$ & $8 \delta_{0}^{(4)}$ & 0.36464934 & 1.3863922 & 0.88604978 & \\
\hline$\frac{1}{2} \oplus \frac{3}{2}$ & $4\left(\delta_{0}^{(4)}-\delta_{2}^{(4)}\right)$ & 0.47382047 & 0.41770663 & 1.0397208 & 0.63630800 \\
\hline $0 \oplus \frac{1}{2}$ & $\notin \mathbb{Q}$ & 0.40013109 & 0.54670471 & 1.1686609 & 0.84652598 \\
\hline $0 \oplus \frac{3}{2}$ & $\notin \mathbb{Q}$ & 0.44457252 & 0.55636026 & 1.1686609 & 0.84694306 \\
\hline $0^{\prime} \oplus 0 \oplus \frac{1}{2}$ & $\notin \mathbb{Q}$ & 0.48094569 & 0.57807813 & 1.2520659 & 0.62795992 \\
\hline & & $3 \times 3 \times 4(\mathrm{~h})$ & $3 \times 4 \times 4(\mathrm{p})$ & & \\
\hline $0^{\prime} \oplus 2^{\prime}$ & $2 \delta_{0}^{(1)}$ & 0.61935581 & 0.81580656 & & \\
\hline
\end{tabular}

Table 3: The site entropy estimated numerically. $L_{1} \times L_{2}$ is the size of a layer while (p) and (h) mean periodic and hard-wall boundary conditions within a layer.

Note that the size of matrix to be diagonalized for $Z_{2}$ is smaller than that for $Z_{2^{\prime} \oplus 2^{\prime}}$.

The exponential growth is severe even after restricting to the $\mathbf{d}=\mathbf{0}$ sector. In order to increase $L_{1} L_{2}$ as much as possible, I have further decomposed $T_{\mathbf{0}}$ according to the eigenvalue of shift (lattice momentum) operator for the periodic boundary case where the translational symmetry is present. I have looked at the zero-momentum sector $T_{\mathbf{0}}{ }^{(0,0)}$ as described in the appendix A. It is quite natural to expect that the largest eigenvalue lies there.

By this decomposition, the dimensionality of the eigenproblem is reduced, at most, by $\left(L_{1} L_{2}\right)^{-1}$. As a drawback, the matrix $T_{\mathbf{0}}{ }^{(0,0)}$ becomes less sparse than the original $T_{\mathbf{0}}$.

With all these effects combined, some improvements in both the memory usage and the computational time are observed and the analysis of larger systems becomes possible for the periodic boundary case, as seen in Tables 2 and 3 .

\section{Discussions}

In the comparison between the periodic and the hard-wall boundary conditions, one notices that the periodic case has always larger site entropy. This is because loops which wind non-trivially in the horizontal directions are allowed in the periodic case and many of them contribute to the sum because $A(L)=0$.

Unfortunately, information in the thermodynamic limit $L_{1}, L_{2} \rightarrow \infty$ is out 
of reach in the present analysis due to the limitation of computational resources. I suppose it will be available in the future research, for the numerical works in the present study have been carried out on only modest workstations.

For the study of criticality, ref. [12], where random walks with the weight in Fig.2 are studied, is quite suggestive. It is reported that Euclidean symmetry is not always recovered even in the continuum limit.

I discuss relations with combinatorial problems below.

\subsection{Even and odd number of loops of a specific type}

For most allowed values of $J$, the loop fugacity takes both positive and negative values. Some interesting combinatorial information is encoded in these model.

For example, the linear combinations $\frac{1}{2}\left(Z_{0^{\prime}} \pm Z_{2^{\prime}}\right)$ counts the number of loop configurations such that there are even (odd) number of loops for which $\frac{2}{\pi} A(L) \equiv 1 \bmod 2$, e.g.,

$$
\frac{1}{2}\left(Z_{0^{\prime}} \pm Z_{2^{\prime}}\right)=\left\{\begin{array}{l}
\sum_{c \in \mathcal{C}_{\text {even }, \frac{1}{2}}} 1, \\
\sum_{c \in \mathcal{C}_{\text {odd }, \frac{1}{2}}} 1
\end{array}\right.
$$

where $\mathcal{C}_{\text {even(odd), } a}$ is the subset of $\mathcal{C}$ and is defined by the following properties.

- $c \in \mathcal{C}_{\text {even(odd), } a}$ contains even (odd) number of loops with $A(L) / a \pi \equiv 1$ $\bmod 2$.

- All other loops in $c \in \mathcal{C}_{\text {even(odd) }, a}$ satisfy $A(L) / a \pi \equiv 0 \bmod 2$.

Similarly, the quantities $\frac{1}{2}\left(Z_{0^{\prime} \oplus 2^{\prime}} \pm Z_{1}\right)$ and $\frac{1}{2}\left(Z_{0^{\prime} \oplus 2^{\prime} \oplus 1} \pm Z_{\frac{1}{2} \oplus \frac{3}{2}}\right)$ are interpreted as the sums over $\mathcal{C}_{\text {even(odd), } 1}$ and $\mathcal{C}_{\text {even(odd), } 2 \text {. }}$

In the limit $L_{3} \rightarrow \infty$ studied in section 3 , one of the two $Z$ 's dominates the sum, the one with the larger leading eigenvalue. In finite geometries, both term contribute to yield an exact number.

\subsection{Hamiltonian cycle}

A Hamiltonian cycle on a lattice is a closed self-avoiding walk which visits every site once and only once. Though it can be enumerated by taking $n \rightarrow 0$ limit of the fully packed loop model $Z_{\text {loop }}\left(n, x^{-1}=0\right)$, no way to construct a local transfer matrix has been known for $n=0$ in three dimensions. Thus the enumeration of Hamiltonian cycles on three-dimensional lattices has been a very difficult problem.

The model I propose in this paper can be regarded as a step forward to such a construction. In the models $Z_{0^{\prime} \oplus 2^{\prime}}, Z_{0^{\prime} \oplus 2^{\prime} \oplus 1}$ and $Z_{0^{\prime} \oplus 2^{\prime} \oplus \frac{1}{2} \oplus 1 \oplus \frac{3}{2}}$, the fugacity is set zero for families of loops. This is, however, achieved by paying the cost of having larger $n$ for other families of loops. Within the present construction, loops with $A(L) \equiv 0 \bmod 4 \pi$ cannot have weights different from the number of possible link states. Thus the partition function listed above serve only as a very loose upper bound for the entropy of Hamiltonian cycles. 


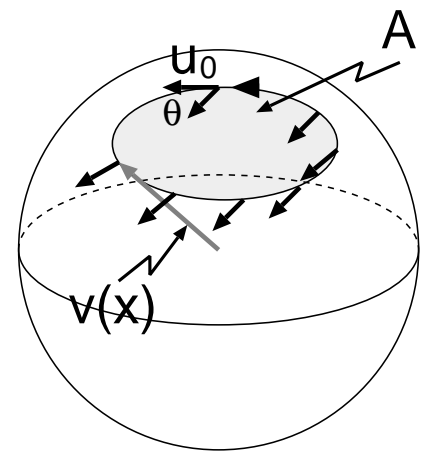

Figure 3: When $\mathbf{u}_{0}$, the tangent vector at $\mathbf{v}\left(\mathbf{x}_{0}\right)$, is parallel-transported along the trajectory on $\mathrm{S}^{2}$, it receives holonomy whose magnitude (angle $\theta$ ) is equal to the oriented area $A(L)$ the path encloses.

The problem of construction of a local transfer matrix to enumerate Hamiltonian cycles on three-dimensional lattices still remains open.

\subsection{Mapping to ribbon configurations}

The oriented area in the definition (3) has a nice geometric interpretation as holonomy. The tangent vector $\mathbf{v}(\mathbf{x})$ moves along a trajectory on $\mathrm{S}^{2}$. Let the unit vector tangential to this trajectory at $\mathbf{v}\left(\mathbf{x}_{\mathbf{0}}\right)$ be $\mathbf{u}_{\mathbf{0}}$. (Fig. 3). Consider the parallel transport (in the sense of riemannian geometry) of the tangent vector $\mathbf{u}_{0}$ along the trajectory $\mathbf{v}(\mathbf{x})$ on $\mathrm{S}^{2}$.

When $\mathbf{u}_{0}$ is transported back to $\mathbf{v}\left(\mathbf{x}_{0}\right)$, it gains some holonomy ( the angle $\theta$ in Fig. 31). This holonomy angle is given by the integration of the scalar curvature of $\mathrm{S}^{2}$ over the domain encircled by the trajectory and is proportional to the oriented area $A(L)$. In the real space $\mathbb{Z}^{3} \subset \mathbb{R}^{3}$, the holonomy described above is nicely kept track of by broadening the loop segment to a 'ribbon' with the distinction of the right and the reversed sides. The parallel transportation can be recasted as a rule of bending ribbons on sites, which is shown in Fig.A. Among the ribbon loop configurations shown in Fig., the partition sum $Z_{\text {loop }}$ with fugacity $n=\delta_{0}^{(2)}, \delta_{0}^{(1)}$ and $\delta_{0}^{\left(\frac{1}{2}\right)}$ receives contribution from $\{(\mathrm{a})\},\{(\mathrm{a}),(\mathrm{b})\}$, and $\{(\mathrm{a}),(\mathrm{b}),(\mathrm{c})\}$, respectively.

Thus, for $n=4 \delta_{0}^{(2)}$, the sum in (3) is over ribbon loop configurations without mismatch for ribbons that have the right and reverse sides. In that interpretation, the coefficient four is naturally explained as the number of directions the right side faces. Therefore, $Z_{\text {loop }}\left[n=4 \delta_{0}^{(2)}\right]\left(x^{-1}\right)$ is nothing but the generating function of the number of allowed ribbon configurations.

\footnotetext{
${ }^{4}$ This observation clearly indicate a simple way of constructing transfer matrices for these
} 


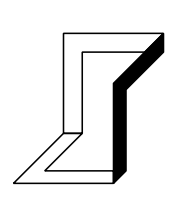

(a)

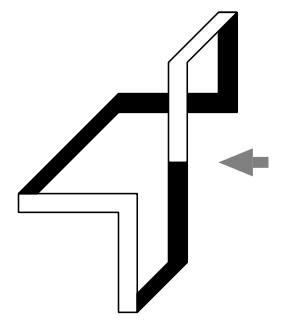

(b)

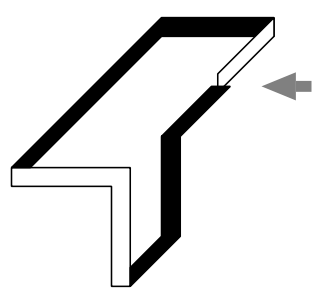

(c)

Figure 4: Examples of allowed ways of bending ribbons at sites (except for the ones indicated by gray arrows) The holonomy is accumulated at the sites indicated by gray arrows. The right and the reverse sides of ribbons are represented by white and black colors.

Similarly, the partition sum for $n=2 \delta_{0}^{(1)}$ can be interpreted as the sum over the configurations of ribbons without the distinction between the right and the reverse sides, while in the case $n=\delta_{0}^{\left(\frac{1}{2}\right)}$, the loop segment is just a chord. This interpretation suggests that (3) may be regarded as a model of polymers with various partially broken axial symmetry by, for instance, the presence of side chains.

\subsection{Comparison with transfer matrices in the connectivity basis}

The connectivity basis 20, 21 is very powerful in that one can always write a transfer matrix for a loop model with respect to it. It is, however, not local one. The fundamental degrees of freedom are not the link variables.

It has been very useful for numerical calculation in two-dimensions. In three dimensions and higher, however, the size of connectivity basis grows considerably because of the lack of the planarity constraint. It is not clear if it is effective to perform numerical calculation in this basis.

It is good to have local one even in two dimensions. Namely, it paved the way to the Bethe ansatz solution 22, 23] and the conformal field theoretic description [24. 25] via Coulomb gas representation. It has also been useful for numerical calculations [9, 17]. Thus I have persisted with the idea of local representation of transfer matrix in this work.

fugacity functions. The link variable represents the ribbon which can be placed in four ways. The rule of bending is implemented in the vertex weight. The construction in section 3 , however, has an advantage; the size of the matrix can be reduced by the choice of the sector $\mathbf{d}=\mathbf{0}$. 


\section{Acknowledgments}

I thank S. Tanimura for useful discussions on the geometric phase. I gratefully acknowledge useful conversations with M. Asano, C. Itoi, S. Hikami, K. Minakuchi, J. Suzuki. I thank T. Iwai and Y. Uwano for discussions and for hospitality at Kyoto University, where a part of this work was done.

This work was supported by the Ministry of Education, Science and Culture under Grant 08454106 and 10740108 and by Japan Science and Technology Corporation under CREST.

\section{A Projection to zero momentum subspace}

In this short note, I describe the block-diagonalization of the transfer matrix with respect to the eigenvalues of lattice momentum operators. The zeromomentum subspace and a reduced transfer matrix which acts on it are explicitly constructed .

One works with the $(2 q+1)^{L_{1} L_{2}}$ dimensional space of colored arrow configurations or an eigenspace of the operator $\mathbf{d}$. One considers the matrix elements in the basis $u_{i},(i=1, \ldots, m)$, each of which represents a single arrow configuration e.g., $\uparrow \uparrow \downarrow|\cdots \uparrow| \downarrow$ :

$$
T u_{i}=\sum_{j=1}^{m} T_{i}^{j} u_{j} .
$$

In this natural basis, the matrix $T$ becomes sparse.

Let $S_{1}$ and $S_{2}$ be discrete shift operators in the horizontal directions. Then the vectors

$$
v_{j}=\sum_{a=0}^{L_{1}-1} \sum_{b=0}^{L_{2}-1}\left(S_{1}\right)^{a}\left(S_{2}\right)^{b} u_{j}
$$

are zero-momentum ones.

One classifies the index set as $\{1, \ldots, m\}=\sqcup_{I=1}^{M} V_{I}$ by an equivalence relation $i \sim j \Leftrightarrow v_{i}=v_{j}$. Then $I=1, \ldots, M$ labels the zero-momentum subspace. The $(I, J)$-component of the block matrix is simply

$$
\left(T_{\mathbf{0}}{ }^{(0,0)}\right)_{I}^{J}=\sum_{j \in V_{J}} T_{i}^{j} \quad\left(i \in V_{I}\right)
$$

This procedure is fairy easy to implement in the sparse algorithm.

Evidently, a slight modification of the above procedure enables one to focus on a chosen non-zero momentum subspace. It will be useful for identifying excited states.

\footnotetext{
${ }^{5}$ It may well improve the efficiency just to choose a zero-momentum state as the initial Lanczos vector in the sparse algorithm without explicitly constructing a transfer matrix in the subspace as is done in the text.
} 
It is noted that the above method can be used for the transfer matrix with the seam factor for enumeration of Hamiltonian cycles 17]. One can make the system translationally invariant by distributing the seam factor among all horizontal

links. I have checked that the above prescription works efficiently although the weight becomes system size dependent.

\section{References}

[1] B. Nienhuis, Exact critical point and critical exponents of $\mathrm{O}(n)$ model in two dimensions, Phys. Rev. Lett. 49 (1982) 1062.

[2] E. Domany, D. Mukamel, B. Nienhuis, and A. Schwimmer, duality relations and equivalences for models with $\mathrm{O}(n)$ and cubic symmetry, Nucl. Phys. B190 (1981) 279.

[3] J. Kondev and C. L. Henley, Four-coloring model on the square lattice: A critical ground state, Phys. Rev. B52 (1995) 6628.

[4] H. S. Chan and K. A. Dill, Compact polymers, Macromolecules 22 (1989) 4559.

[5] H. W. J. Blöte and B. Nienhuis, Fully packed loop model on the honeycomb lattice, Phys. Rev. Lett. 72 (1994) 1372.

[6] H. Orland, C. Itzykson, and C. de Dominicis, An evaluation of the number of Hamiltonian paths, J. Physique 46 (1985) L353.

[7] J. Suzuki, Evaluation of the connectivity of Hamiltonian paths on regular lattices, J. Phys. Soc. Japan 57 (1988) 687.

[8] S. Higuchi, Field theoretic approach to the counting problem of Hamiltonian cycles of graphs, Phys. Rev. E58 (1998) 128, cond-mat/9711152.

[9] M. T. Batchelor, H. W. J. Blöte, B. Nienhuis, and C. M. Yung, Critical behaviour of the fully packed loop model on the square lattice, J. Phys. A 29 (1996) L399.

[10] S. K. Nechaev, Statistics of knots and entangled random walks, World Scientific, Singapore, 1996, cond-mat/9812205.

[11] J. L. Jacobsen, On the universality of compact polymers, preprint, condmat/9903132.

[12] M. Asano, C. Itoi, and S.-I. Kojima, Random walk construction of spinor fields on three-dimensional lattice, Nucl. Phys. B448 (1995) 533, hepth/9412215. 
[13] M. Asano, C. Itoi, and S.-I. Kojima, Quantum diffusion process with a complex weight on three-dimensional lattice, in Proceedings to the International Seminar Devoted to the 140th Birthday of Henri Poincaré, Protovino, Russia, 1994, IHPE, hep-th/9410090.

[14] C. Itoi, Smooth paths on three-dimensional lattice, Phys. Rev. Lett. 73 (1994) 3335, hep-th/9406123.

[15] A. M. Polyakov, Fermi-Bose transmutations induced by gauge fields, Mod. Phys. Lett. A3 (1988) 325.

[16] S. Iso, C. Itoi, and H. Mukaida, Geometric description for spinning particles in three dimensions and Chern-Simons-Polyakov theory, Phys. Lett. B236 (1990) 287.

[17] S. Higuchi, Compact polymers on decorated square lattices, J. Phys. A 32 (1999) 3697, cond-mat/9811426.

[18] P. Di Francesco, E. Guitter, and C. Kristjansen, Fully packed $\mathrm{O}(n=1)$ model on random Eulerian triangulations, preprint, cond-mat/9902082.

[19] E. Guitter, C. Kristjansen, and J. L. Nielsen, Hamiltonian cycles on random Eulerian triangulations, Nucl. Phys. B546[FS] (1999) 731, condmat/9811289.

[20] T. G. Schmalz, G. E. Hite, and D. J. Klein, Compact self-avoiding circuits on two-dimensional lattices, J. Phys. A 17 (1984) 445.

[21] H. W. J. Blöte and B. Nienhuis, Critical behaviour and conformal anomaly of the $\mathrm{O}(n)$ model on the square lattice, J. Phys. A 22 (1989) 1415.

[22] R. Baxter, Exactly solvable models in statistical mechanics, Academic Press, London, 1982.

[23] M. T. Batchelor and B. H. W.J., Conformal anomaly and scaling dimensions of the $\mathrm{O}(n)$ model from an exact solution on the honeycomb lattice, Phys. Rev. Lett. 61 (1988) 138.

[24] J. Cardy, Geometrical properties of loops and cluster boundaries, in Fluctuating geometries in statistical mechanics and field theory, edited by F. David, P. Ginsparg, and Z. Zinn-Justin, Les Houches Session LXII, Elsevier, 1994.

[25] J. L. Jacobsen and J. Kondev, Field theory of compact polymers on the square lattice, Nucl. Phys. B532[FS] (1998) 635, cond-mat/9804048. 